\title{
LA BÚSQUEDA DE SOLUCIONES CREATIVAS DESDE LA PRÁCTICA LABORAL EN LA CARRERA EDUCACIÓN PREESCOLAR
}

\author{
The Search for Creative Solutions from labor Practice in Career Preschool \\ Education
}

\author{
Maidelys Rodríguez Álvarez, MsC. \\ Universidad de Sancti Spíritus "José Martí \\ Pérez", Cuba \\ https://orcid.org/0000-0002-1061-9718 \\ ereinoso@uniss.edu.cu
}

\author{
Juana María Vázquez Vázquez, MsC. \\ Universidad de Sancti Spíritus “José Martí \\ Pérez", Cuba \\ https://orcid.org/0000-0003-0304-8301 \\ eddys@uniss.edu.cu
}

\author{
Yudith del Cristo Sosa, MsC. \\ Universidad de Sancti Spíritus "José Martí \\ Pérez", Cuba \\ https://orcid.org/0000-0003-1311-2573 \\ ydelcristo@uniss.edu.cu
}

Palabras claves: Actuación, Creativo, Laboral, Investigativo, Integradora.

Keywords: Performance, Creative, Labor, Investigative, Integrator.
Recibido: 18 de febrero de 2021

Aceptado: 23 de abril de 2021

\section{RESUMEN}

La solución de determinadas tareas en la práctica constituye el punto de partida para la Disciplina Principal Integradora, la cual se apoya en los aportes de las restantes disciplinas de la carrera y las asume en su integración para dar respuesta a las exigencias del quehacer profesional para la Educación Preescolar, asegura el dominio de los modos de actuación esenciales, sin embargo, se hace necesario potenciar el desarrollo de la creatividad en situaciones que se presentan en la práctica educativa. El presente trabajo forma parte de una investigación que se realiza en la universidad, se hace referencia a los fundamentos teóricos y metodológicos acerca del modo de actuación creativo de los profesionales en formación desde el proceso de enseñanza aprendizaje de la Disciplina Principal Integradora.

\section{ABSTRACT}

The solution of certain tasks in practice constitutes the starting point for the Principal Integrative Discipline, which is based on the contributions of the remaining disciplines of the career and assumes them in their integration to respond to the demands of the professional work for the Preschool Education, ensures the mastery of the essential modes of action, however it is necessary to enhance the development of creativity in situations that arise in educational practice. The present work is part of a research carried out in the university, reference is made to the theoretical and methodological foundations about the creative acting of the professionals in training from the teaching process of the Principal Integrative Discipline. 


\section{INTRODUCCIÓN}

Hablar de creatividad educacional en el siglo XXI es integrar en una frase la esencia de este momento crucial de la humanidad. Para ello, hay que humanizar la escuela: al alumno contemporáneo le hace falta aprender a resolver problemas, saber escuchar, organizarse, tener buen humor, analizar críticamente la realidad, transformarla, amar a sus semejantes, tener cultura en el más amplio sentido de la palabra, no restringirla solo a conocimientos; sino implicando a los valores universales del hombre.

Por tanto, es preciso que, desde las aulas, se desarrolle la independencia cognoscitiva, la avidez por el saber, de manera tal que no haya miedo de resolver cualquier situación por difícil que esta parezca.

La creatividad ha sido y es objeto de análisis de muchas disciplinas como la psicología, la sociología, la epistemología, la filosofía, la pedagogía. Diversos son los estudios que centran su atención en el desarrollo de la creatividad en educación, a partir de las particularidades de la actividad pedagógica profesional, las características de la escuela, las estrategias de enseñanza y aprendizaje y el rol del maestro, entre otros aspectos.

En las obras de destacados estudiosos de la creatividad como W. Gordon (1961), A. F. Osborn (1963), S. Parnes (1972), E. P. Torrance (1976), G. Melhorn (1978), E. Bono (1982), S. de la Torre (1987), aparecen aportes interesantes sobre los problemas del desarrollo y educación de la creatividad en la escuela.

Una de las líneas de investigación en las que se han desarrollado diferentes estudios es la relacionada con el desarrollo de la creatividad en los profesores. Autores como A. González (1990-1994), M. D. Córdova (1992), E. Caballero (1993), García (1997), S. Fariñas (1997), M. Martínez Llantada (1998, 2003), R Martínez (1998), I. Daudinot (1999), M. Macías(2003), J. Remedios(2001, 2003, 2005) y N. Calero (2005), entre otros, atienden diferentes aristas de esta línea investigativa; la mayoría coincide en la necesidad de buscar vías que estimulen las potencialidades creadoras de los profesores.

El transformar la educación implica también cambios en la actuación profesional pedagógica, ya que se hace imprescindible que el profesional sea capaz de auto perfeccionarse, instrumentar los cambios necesarios y deseados en su contexto de actuación, pero más aún, que se motive a buscar y proyectar continuamente nuevas alternativas fundamentadas científicamente, que contribuyan a perfeccionar la realidad educativa en la que se desarrolla.

En esta dirección, en la provincia de Sancti Spíritus, se han desarrollado varios proyectos de investigación (2001-2009). En sus resultados se ha profundizado en las características del modo de actuación creativo del docente, lo que permite comprender que el desarrollo de la creatividad requiere que se estimule desde la formación inicial del profesional de la educación.

La referida formación del profesional de la educación es permanente, pero este período de la formación inicial es de suma importancia y es entendida como el proceso de apropiación de conocimientos, habilidades, valores y métodos de trabajo pedagógico que prepara al estudiante para el ejercicio de las funciones profesionales pedagógicas y se expresa mediante el modo de actuación profesional que va desarrollando a lo largo de la carrera (Chirino, M. V., 2003:3).

Es decir, en este período el significado que el estudiante le concede a la profesión se consolida en dependencia de muchos factores, dentro de los que se pueden mencionar las condiciones de sus centros de formación y el sistema de relaciones que establece con los diferentes sujetos y objetos con los que interactúa, para llegar a formar su modo de actuación profesional, desde su práctica donde la creatividad se convierte en aspecto esencial de su modo de actuación.

En tal sentido, Juana María Remedios (2005:10) y Nancy Calero (2005:7) al referirse al modo de actuación profesional creativo precisan que este se expresa mediante: "la ejecución de acciones originales, independientes y flexibles, acorde con las exigencias educativas, a partir de una elevada motivación profesional".

Todo lo antes planteado apunta a la inserción de la formación del profesional en el medio laboral y cómo esta debe garantizar no solo la adquisición de conocimientos teórico-prácticos y el desarrollo de habilidades profesionales, sino también, la búsqueda de soluciones creativas en su medio y para su medio.

De esta forma se puede comprender cómo la práctica juega un papel importante en el proceso creador; pues el profesor en formación puede crear mediante el trabajo nuevos medios y desarrollar los procesos asociados a la creatividad a partir de sus propósitos, intereses y condiciones: "Sólo desde los problemas que aparecen en las situaciones complejas e irrepetibles del aula, la escuela, la comunidad, puede hacerse significativo y útil para el estudiante, el conocimiento académico teórico." (García, G. y Addine, F. 2002: 9). 
En el Plan del Proceso Docente de la carrera Licenciatura en Educación Preescolar, la Disciplina Principal Integradora (DPI) Formación Laboral Investigativa, favorece el desarrollo de los procesos asociados a la creatividad en la actividad pedagógica.

La concepción del desarrollo de la creatividad a través de la Disciplina Principal Integradora Formación Laboral Investigativa en la carrera de Educación Preescolar, presenta una manifestación práctica incipiente con respecto a la manera particular del desarrollo del proceso de enseñanza-aprendizaje para lograr la integración de los contenidos que permite la formación de los modos de actuación del futuro profesional caracterizado por su creatividad; sin embargo se aprecia un vacío teórico al respecto que exige una respuesta científica de rigor.

El presente trabajo forma parte del proyecto investigativo que se realiza en la UNISS, el cual se titula: "La formación didáctica del profesional universitario para dirigir la solución de problemas", se deriva además de la investigación que realiza la autora principal como parte de su formación doctoral, tiene el objetivo de exponer los fundamentos teóricos y metodológicos acerca del modo de actuación creativo de los profesionales en formación de la carrera en educación preescolar desde el proceso de enseñanza aprendizaje de la Disciplina Principal Integradora.

\section{DESARROLLO}

\section{Referentes teóricos acerca del proceso de enseñanza aprendizaje de la Disciplina Principal Integradora.}

El eje central de una concepción pedagógica, sirve de fundamento a los actuales planes de estudio y a su perfeccionamiento, descansa - como es sabido - en la filosofía de aprender pensando, investigando y haciendo. En un ámbito más reducido, constituye el modo más idóneo de cumplir con lo que desde hace aproximadamente una década viene orientando nuestro Partido, la formación de un profesional de perfil amplio, capaz de aplicar oportunamente los conocimientos adquiridos en la Universidad.

Al consultar la literatura especializada en didáctica, se aprecia que los diferentes autores fundadores de esta ciencia, daban importancia significativa a la categoría enseñanza.

En el análisis realizado a la obra de J. A. Comenio (1983) se identifican varios elementos de su contenido que se relacionan con las bases psicológicas del proceso didáctico: la didáctica como el arte de enseñar y aprender, la exigencia de que la enseñanza debe ser atractiva y con agrado, la necesaria solidez de los contenidos que se aprenden, el deseo de saber de los estudiantes con elemento básico, la apelación al entendimiento y a los sentidos del aprendiz, el carácter procesal del aprendizaje y el basamento en procesos psíquicos fundamentales, tales como la atención, la memoria y el lenguaje.

Otros pedagogos más tarde enriquecieron estas valiosas concepciones sobre las enseñanzas teóricas de Comenius entre los que se encuentran, Juan Jacobo Rosseau (1712-1978) quien colocó en la base de su concepción de la enseñanza, los intereses de los escolares; Enrique Pestalozzi (1746-1827) que atribuyó gran valor a la psicología infantil como fundamento de la enseñanza ; Juan Federico Herbart (1776-1841) que propuso una serie de pasos formales de base a la estructuración de su concepción de enseñanza a modo de orientación a los profesores sobre la conducción de este proceso para el aprendizaje de los alumnos.

Un lugar importante lo ocupó K. D. Ushinski (fines de siglo XVIII, inicios del XIX) que destacó entre sus consideraciones el papel del maestro como conductor de la enseñanza y a los alumnos en su actividad de aprendizaje de conocimientos y habilidades, transmitido por este.

No es posible señalar aspectos importantes acerca de los procesos de enseñanza y aprendizaje sin hacer referencia a algunas ideas expresadas por eminentes pedagogos cubanos, que sin dudas han enriquecido la concepción acerca de estos procesos, las cuales se constituyeron en fuertes críticas al escolasticismo, el memorismo mecánico y el dogmatismo característicos de la escuela cubana de los siglos XVIII y XIX. Entre varios pensadores de esta etapa se destaca Félix Varela, quien enfatizó en la necesidad de enseñar al hombre a pensar desde sus primeros años, a quitarle los obstáculos de que piense.

Por otra parte, todos los esfuerzos de José de la Luz y Caballero estuvieron dirigidos a lograr en los alumnos durante su aprendizaje el interés por la investigación, así como la independencia en la adquisición del conocimiento; la importancia de la formación de jóvenes críticos que no repitan, ni aprendan de memoria. En el ideario pedagógico de José Martí y Pérez se encuentra entre otras ideas, el resumen de todo lo positivo expresado con anterioridad con respecto a la enseñanza y al aprendizaje en nuestro país. 
Mucho más reciente, el autor Lotear Klingberg (1972:220), uno de los representantes de la didáctica contemporánea, ya considerada como ciencia, valora entre sus ideas que la enseñanza está determinada en gran medida por el maestro y su actividad, la instrucción y la educación, señalando que la enseñanza es siempre un proceso de enseñanza y aprendizaje, en el que se relacionan entre sí maestro y alumnos.

Al analizar el contenido de algunas de las ideas expresadas por los diferentes autores acerca de la enseñanza, tanto en unos como en otros se aprecia el significado de esta en su papel rector, pero siempre conducente del proceso de aprendizaje de los alumnos, y aún más como última idea en Klingberg conducente como proceso, a la formación de la personalidad.

Lo importante expresado en el ideario de estos pensadores es que se trata de un proceso donde están presentes la enseñanza y el aprendizaje, en el cual el papel conductor de guía lo tiene el maestro. Además, en correspondencia con lo planteado anteriormente el mencionado autor le concede especial atención a la unidad de la enseñanza y el aprendizaje.

En la actualidad existen varias concepciones, teorías y modelos en el campo de la didáctica, de acuerdo con los criterios propuestos por diferentes autores. El reflejo de estos procesos, en la literatura pedagógica y psicológica más vinculada a una concepción desarrolladora, tiene su centro en las ideas de Vigotsky.

La teoría de Vigotsky está basada principalmente en el aprendizaje sociocultural de cada individuo y, por lo tanto, en el medio en el cual se desarrolla. Él consideraba el aprendizaje como uno de los mecanismos fundamentales del desarrollo, en su opinión, la mejor enseñanza es la que se adelanta al desarrollo. En el modelo de aprendizaje que aporta, el contexto ocupa un lugar central. La interacción social se convierte en el motor del desarrollo.

Vigotsky introduce el concepto de zona de desarrollo próximo que es la distancia entre el nivel real de desarrollo y el nivel de desarrollo potencial. Para determinar este concepto hay que tener presentes dos aspectos: la importancia del contexto social y la capacidad de imitación.

Diferentes investigaciones realizadas en los últimos años en nuestro país (Margarita Silvestre y Pilar Rico, 1998) (Zilberstein, 1999) y en otras partes del mundo, afirman que el objeto de estudio de la Didáctica es el proceso de enseñanza aprendizaje. Este proceso transcurre fundamentalmente en las asignaturas que integran el currículo, aunque se debe considerar que hoy este proceso no deberá desarrollarse sólo en los salones de clases, sino incluir el trabajo en el hogar y la comunidad; su propósito fundamental es contribuir a la formación integral de los estudiantes con orientaciones valorativas expresadas en sus formas de sentir, pensar y actuar, que estén en correspondencia con el sistema de valores de cada sociedad.

El proceso de enseñanza aprendizaje constituye la vía mediatizadora esencial para la apropiación de conocimientos, habilidades, hábitos, normas de relación, de comportamientos y valores, legados por la humanidad, que se expresan en el contenido de enseñanza, en estrecho vínculo con el resto de las actividades docentes y extradocentes que realizan los estudiantes (Zilberstein Toruncha, J: 1999).

La investigadora Doris Castellanos y otros (2000) en su libro Para promover un aprendizaje desarrollador se refiere al aprendizaje desarrollador a aquel proceso que promueve el desarrollo óptimo, multifacético, de la personalidad de niños/niñas, adolescentes y jóvenes, que garantiza en los individuos la apropiación activa y creadora de la cultura, propiciando el desarrollo de la autonomía, y la autodeterminación, en íntima armonía con los procesos de socialización, compromiso y responsabilidad social.

Las autoras Pilar Rico y Margarita Silvestre (2000:3) hacen referencia al proceso de enseñanza aprendizaje como un sistema integrado, consciente del papel protagónico del alumno en dicho proceso. En este enfoque se revela como característica determinante la integración entre lo cognitivo y lo afectivo, lo instructivo y lo educativo, como requisitos psicológicos y pedagógicos esenciales.

El proceso de enseñanza-aprendizaje es un contexto de interrelación e intercomunicación de varios sujetos, ya que se dan en un grupo en el cual el maestro ocupa un lugar de gran importancia como educador que lo organiza y conduce, pero en el que no se logran resultados positivos sin el protagonismo, la actitud y la motivación del alumno (Rico, Pilar 2004: 41).

Las definiciones antes mencionadas tienen puntos de contacto, pues consideran que en este proceso existe una relación dialéctica entre el profesor que enseña y el estudiante que aprende, los cuales se diferencian por sus funciones; el profesor debe estimular, dirigir y controlar el aprendizaje de manera tal que el alumno sea participante 
activo, consciente en dicho proceso, teniendo en cuenta como premisa esencial la relación de lo afectivo y lo cognitivo, así como lo instructivo y educativo .

La autora Addine F, F en su libro La Didáctica General y su enseñanza en la Educación Superior Pedagógica(2013:14) define al proceso de enseñanza aprendizaje como un proceso que debe estudiarse e investigarse desde una dimensión proyectiva, que incluya su diseño, ejecución, evaluación y oriente sus resultados a lo personal y social, a partir de un presente diagnosticado hasta llegar a un futuro deseable, donde se reconozca la multilateralidad de interrelaciones y la heterogeneidad de los participantes.

La concepción que se sostiene parte de valorar el qué, el cómo y el para qué de la unidad de la teoría y la práctica desde un marco de análisis que posibilite un proceso de enseñanza y aprendizaje desarrollador. Las autoras de esta investigación asumen la definición dada por la Dra.C Fátima Addine, pues hace referencia de forma integral el desarrollo de la personalidad desde la teoría y la práctica, siendo este un elemento fundamental en el profesional universitario al que aspiramos formar.

\section{El diseño de la Disciplina Principal Integradora Formación Laboral Investigativa.}

La formación del profesional se desarrolla en distintos niveles estructurales, desde la tarea docente, los temas, las asignaturas, las disciplinas hasta llegar al nivel de carrera. Sin embargo, estas partes no se presentan aisladas y ninguna constituye la suma de otras. Cada tema o asignatura, presenta contenidos, modelaciones o abstracciones de la realidad que preparan al estudiante en la esencia de lo que se estudia, la sistematización paulatina de los contenidos y de sus relaciones de manera que estos se vayan acercando cada vez más a la realidad circundante y compleja que presenta su futura profesión.

Entre los niveles estructurales que como cualidad aparecen en el proceso de formación del profesional, se destaca uno que para las autoras de esta investigación tiene singular importancia: la disciplina. Pueden establecerse, entre otras clasificaciones, dos tipos de disciplinas: aquellas que les permiten a los estudiantes profundizar en el objeto de cada una de las ramas de la ciencia (disciplinas derivadoras) y las que enfrentan al estudiante con los contenidos de la actividad profesional propiamente dicha, una vez egresado y que reflejan la realidad en su totalidad globalizadora (Disciplina Principal Integradora).

Concebir el modo de actuación creativo en los profesionales en formación de la carrera Licenciatura en Educación especialidad Preescolar no resulta posible actualmente fuera del contexto de la disciplina Formación Laboral Investigativa como principal integradora.

El término disciplina integradora no tiene una acepción común en todos los contextos. De manera bastante frecuente se utiliza para denotar la integración de conocimientos de áreas de cuyo objetivo es proporcionar mejores productos, procesos y sistemas o bien cuando se afirma que las contribuciones desde otras disciplinas científicas son imprescindibles.

En la realidad universitaria, la disciplina integradora ha sido trabajada en el orden metodológico por diferentes autores tales como: Alonso, I. (2000), González, H. (2000), Fernández, R (2000), Fuentes González, H C. (2002), Ortiz, A. (2002), Álvarez, I (2002), Martín, F. (2002); Balbis, E. (2002), (López, 2003), sin embargo, en los aspectos teóricos y de diseño curricular no aparece muy referenciada. Álvarez de Zayas, (1998) establece que la disciplina integradora es aquella que tiene como objeto de estudio el objeto de trabajo del egresado, el ejercicio de la profesión.

Los trabajos más conocidos sobre diseño de una disciplina son los de Álvarez V., I.B. (1996); Fuentes González, H C. (1998); Cruz Baranda., S. (1998), Malagón Hernández (1998), Ferreira Lorenzo (2005), centrando su importancia en el objeto de trabajo del futuro profesional.

Las autoras de esta investigación asumen que la disciplina Formación Laboral Investigativa, organizada en forma de sistema debe dar respuesta a las crecientes necesidades y perspectivas del desarrollo económico y social del país, debe servir como elemento de cohesión entre el resto de las disciplinas, de ahí su carácter integrador.

La disciplina Formación Laboral Investigativa vinculada permanentemente con la realidad social, económica, cultural, educativa e investigativa del entorno que rodea a la Universidad. Su contenido refleja, además del sistema de conocimientos particulares, las relaciones que se dan entre los hombres en el trabajo, las relaciones humanas, la comunicación, el vínculo con organizaciones, en fin, los aspectos sociológicos de la actividad del egresado.

Al organizar el contenido de la disciplina Formación Laboral Investigativa debe interrelacionar aquellos contenidos recibidos de las diferentes disciplinas del plan de estudio y posibilitar que el estudiante se apropie del objeto de su trabajo mediante la solución de problemas de la práctica social. En ella está presente no solo el estudio como exponente 
de lo académico y el trabajo como representación de lo laboral, sino también el método de la investigación científica, por eso su nivel de asimilación parte desde lo productivo hasta lo creativo y su evaluación es problémica.

Por otra parte, el objetivo de la disciplina Formación Laboral Investigativa y el objetivo del modelo del especialista están interrelacionados, y cuando un estudiante cursa y aprueba esta disciplina, está en condiciones de trabajar como profesional en las esferas o contextos de actuación requeridos, pues se ha apropiado de los objetivos generales de la carrera.

Esta disciplina permite consolidar la formación académica del estudiante de la especialidad de Educación Preescolar, con habilidades, aptitudes, críticas reflexivas y constructivas propias de su actividad como profesional, generando conciencia, sentido de responsabilidad y vivencia profesional, cooperación y compromiso social, como complemento del proceso de formación universitario.

Tomando como referencia el estudio realizado por el Dr. Pedro Horruitiner Silva (2009) donde refiere que, de todas las disciplinas de una carrera, la universidad cubana identifica una en particular que, por su importancia, es la columna vertebral del proceso de formación: la disciplina principal integradora. Es una disciplina sui generis, que lejos de obedecer a la lógica de una o de varias ciencias, responde a la de la profesión. Ella se apoya en los aportes de las restantes disciplinas de la carrera y las asume en su integración para dar respuesta a las exigencias del quehacer profesional, asegurando el dominio de los modos de actuación esenciales de ese profesional.

En la mayor parte de las carreras se desarrolla desde el primer año y concluye con el trabajo de diploma. De tal modo, está presente durante todo el proceso de formación, ocupando en la mayoría de los casos alrededor de la tercera parte del tiempo lectivo total de la carrera. Es la mayor de todas las disciplinas, además de ser la más importante.

El objetivo que persigue la Disciplina Principal Integradora, es propiciar una mayor interacción entre las diferentes formas de enseñanza, una más acentuada interrelación entre la teoría y la práctica, integrando los siguientes componentes: laboral, investigativo y académico., independientemente del valor que cobra en esta integración la formación ideopolítica y la extensión universitaria, de esta manera se logra un mayor control del aprendizaje. La concepción de la Disciplina Principal Integradora tiene a su vez un enfoque interdisciplinario.

Horruitiner Silva plantea además que el contenido fundamental de la disciplina principal integradora es investigativolaboral. Integra toda la actividad laboral realizada por los estudiantes, así como todos los trabajos científicos estudiantiles. De ese modo se ha concebido en la mayoría de las carreras, pero ello no significa que el problema de su diseño esté totalmente resuelto. Por el contrario, dada su novedad y complejidad pedagógicas es un aspecto donde la educación superior cubana labora intensamente para su perfeccionamiento.

En particular resulta necesario comprender su papel en la labor educativa. Ya se ha hablado de la importancia de incorporar determinados valores al proceso de formación de los profesionales que permitan su exitoso desempeño en la sociedad. Esta disciplina, por sus peculiaridades, ofrece las mayores posibilidades para el desarrollo en los estudiantes de esas cualidades, ya que se realiza en contacto directo con el quehacer profesional. Es precisamente allí, en el contexto de su propia actividad laboral, donde mejor se puede evidenciar un comportamiento adecuado.

La Disciplina Principal Integradora manifiesta la tendencia a la existencia de contenidos multi o polidisciplinarios, que no es más que la asociación de conceptos de otras disciplinas en virtud de un proyecto o de un objeto que le es común en un momento dado, es decir que están en profunda interacción para tratar de concebir este objeto o proyecto; que hagan uso de la lógica o métodos de trabajo de varias ciencias ya estudiadas, (Morin, 1990) o que se presenten por primera vez en el contexto de tecnologías concretas, demostrándose la dialéctica de lo multidisciplinario a lo disciplinario.

El aprendizaje en esta disciplina va de niveles de asimilación de lo productivo a lo creativo debido a que la profesión atiende más a la ejecución, a la tecnología, a la solución de problemas productivos y la ciencia, al enriquecimiento del saber humano, a su creación. Aquí no hay una barrera evidentemente definida pues la profesión alcanza niveles de asimilación productivos, o sea, ante situaciones nuevas el hombre resuelve haciendo uso de lo que sabe.

En resumen, la disciplina garantiza la formación de los modos de actuación del profesional, se desarrolla de primero a quinto año, abarca, como promedio, la tercera parte del total del currículo, su contenido esencial es investigativo-laboral y a ella se subordinan las restantes disciplinas. (Horruitiner Silva, 2009).

La dialéctica académico - laboral (estudio - trabajo) se da muy fehacientemente en esta disciplina ya que refleja la vida, el trabajo, pero no se debe identificar con la relación teoría - práctica porque son aspectos cualitativamente distintos y 
ambos son imprescindibles. Por tanto, las relaciones teoría - práctica como estudio - trabajo son imprescindibles para la formación.

La investigación científica con su metodología propia es la expresión más alta de la habilidad que debe dominar el estudiante en esta disciplina; ya que lo investigativo es consustancial de lo académico y de lo laboral, por lo que debe estar presente.

\section{La Disciplina Principal Integradora Formación Laboral Investigativa en la carrera de Educación Preescolar.}

La disciplina integra los contenidos de todas las disciplinas del Plan del Proceso Docente para la Educación Preescolar. Permite en la práctica la implementación de los modos de actuación del profesional donde se concreta la relación de la teoría con la práctica y se consolidan los conocimientos teóricos y prácticos y el desarrollo de las habilidades profesionales.

En esta disciplina es fundamental trabajar los contenidos desde lo teórico, lo metodológico, lo práctico y lo investigativo, de manera tal que esto permita penetrar en la esencia del objeto de la profesión, es decir, el proceso educativo, concebido como el proceso sistémico de transmisión y apropiación de la cultura en las modalidades curriculares de la Educación Preescolar en función del encargo social, con la finalidad de formar una personalidad integral en los futuros ciudadanos.

La disciplina se sustenta en el principio estudio- trabajo, la actividad práctica como fuente de estimulación motivacional, el vínculo teoría-práctica como expresión del dominio instrumental y metacognitivo, el carácter de sistema de la dirección del proceso educativo y las características de la Educación Preescolar.

Los profesionales en formación de la carrera Educación Preescolar a través de la Disciplina Principal integradora y de las otras disciplinas que conforman el Plan del Proceso docente contribuyen a que el educador de la primera infancia en su actividad profesional le dé solución a los problemas relacionados con la dirección del proceso educativo que dirige para la educación integral de las niñas y los niños de cero a seis años en las dos modalidades del currículo y la coordinación del sistema de influencias educativas que deben ejercer conjuntamente con la familia y a otros agentes educativos.

La experiencia acumulada en la formación de los educadores que requiere este subsistema, unida al desarrollo alcanzado por cuadros, docentes y profesores, permiten acometer el perfeccionamiento de la carrera de acuerdo con los objetivos y principios planteados para la preparación inicial y permanente de los profesionales que necesita el desarrollo científico técnico y social del país.

\section{El modo de actuación creativo de los profesionales en formación.}

Educar en la creatividad es educar para el cambio y formar personas ricas en originalidad, flexibilidad, visión futura, iniciativa, confianza, amantes de los riesgos y listas para afrontar los obstáculos y problemas que se les van presentado en su vida escolar y cotidiana, además de ofrecerles herramientas para la innovación. (Betancourt, J., 1999: 4)

Las palabras de este autor sirven de guía para el estudio que se realiza en relación con la creatividad al pretender profundizar en algunas de las problemáticas sobre el tema en cuestión.

En la literatura revisada, lo relacionado con el modo de actuación, es que su formación se asocia a las acciones que ejecuta la persona en una actividad, por ello, para su explicación resultan interesantes los fundamentos sobre la teoría de la actividad y la comunicación expresados en las obras de L. S. Vigotski, A. N. LeLeontiev, A. R. Luria, N. Talísina, Y. U. Galperin, J. Lompscher, A. K. Markova, V. V. Davidov, R. Bermúdez y L. García.

La actividad pedagógica profesional no está compuesta por una secuencia de acciones fijas: su estructura está dada en general por determinada secuencia de acciones o complejo de acciones, o ambos inclusive, que se superponen o interrelacionan de diversas formas.

En resultados científicos de cubanos que se han dedicado al estudio de esta temática aparecen diferentes definiciones del concepto modo de actuación o modos de actuación del profesional pedagógicos.

Son las formas históricamente condicionadas de desempeñarse el docente, constituidas por el conjunto de métodos y estados para la comunicación y la actividad pedagógica, las cuales revelan un determinado nivel de desarrollo de sus habilidades y capacidades, así como de constructos, rutinas y esquemas y modelos de actuación profesional. (García, L., 1996: 20) 
El colectivo de investigadores del proyecto Vías que contribuyen a transformar los modos de actuación y a desarrollar las potencialidades creadoras de los docentes entiende el modo de actuación de estos en el ejercicio de la actividad pedagógica profesional como el sistema de acciones, para la comunicación y la actividad pedagógica, que modela la ejecución del docente en un determinado contexto de actuación, las cuales revelan el nivel de desarrollo de sus conocimientos, habilidades, capacidades, potencialidades creadoras y le sirve como medio para autoperfeccionarse. (Remedios, J., 2001: 13).

Por su parte M. Castillo en su estudio referido al modo de actuación del profesional de la educación en formación, citado por Addine (2001: 11) expresó: Se puede hablar de modo de actuación profesional, cuando se demuestra por parte del estudiante el dominio pleno de los conocimientos, habilidades, valores y capacidades más generales del objeto de la profesión, que permiten al mismo la aprehensión del método para su actuación profesional, imbricándose en un sistema de acciones generalizadoras de su actividad, adaptables a variadas formas y contextos, tributando la interacción de las mismas a la conformación de cualidades y rasgos distintivos de la personalidad, lo que nos permite identificar la especificidad del objeto y el encargo social de una profesión, y poder discernir entre ese profesional y otro.

En correspondencia con los elementos hasta aquí expuestos se define el concepto de modo de actuación creativo para el profesor en formación como: la ejecución de acciones originales, independientes y flexibles, acorde con las exigencias educativas, a partir de una elevada motivación profesional, mediante las que se expresa el desarrollo y actualización de los conocimientos y habilidades profesionales.

Indudablemente, el desarrollo de la sociedad en la actualidad requiere de hombres abiertos al cambio, listos a resolver cuanto problema surja con un criterio alto de responsabilidad moral.

A la luz del análisis realizado, se precisa que la motivación profesional, la flexibilidad, la originalidad y la independencia se manifiestan en la actuación del educador en una interrelación que asume un carácter dialéctico, multifactorial, plurideterminado, por lo que su estudio metodológico requiere de una concepción integradora que evite atomizar cualquiera de estos aspectos.

Cuando el educador jerarquiza los motivos profesionales en correspondencia con las particularidades de la actividad pedagógica y es capaz de elaborar su proyecto de actuación profesional, y lo asume, está en condiciones favorables para crear productos originales, orientarse en situaciones nuevas, elegir vías para mejorar su propio desarrollo individual y argumentar sus resultados en correspondencia con las particularidades del contexto, así como, personalizar el cambio educativo a partir del para qué del cambio, el porqué, el qué, el cómo, el con qué y qué ha logrado.

Una exigencia del Modelo del Profesional de la carrera de Educación Preescolar es el proceso de formación, el cual debe lograr que los egresados de esta carrera sean capaces de transmitir y educar sentimientos, cualidades morales, volitivas, hábitos culturales y sociales, y la necesidad de la formación de vivencias afectivas y normas de comportamiento social en correspondencia con el contexto socio-histórico y multifactorial de la sociedad. En correspondencia con esto, las autoras le atribuyen gran importancia a las actividades y tareas que desarrollan las estudiantes desde la disciplina Principal Integradora como parte de su formación profesional para que al desarrollar la práctica pedagógica manifiesten modos de actuación creativos.

En tal sentido se puede afirmar tal y como lo plantea nuestro héroe nacional; "Sólo el maestro creador, que ame la profesión puede hacer posible que la inteligencia no sea atributo de pocos, sino que se convierta en patrimonio de todos y una posibilidad para el progreso social." (2004:83).

La formación del profesional de la educación significa preparar un educador que esté en capacidad de cumplir las funciones profesionales sustantivas a que se ha de enfrentar. Para lograrlo se debe desarrollar entonces, un proceso de formación que tenga en cuenta la aspiración, dada por el modelo del profesional, el contenido, las acciones y los escenarios de formación, concretados y articulados de manera sistémica en un modelo de formación sobre la base de determinados fundamentos teóricos, para alcanzar un producto definido por un modelo de actuación, elemento que se convierte en eje conductor de la investigación que se realiza, el lograr un equilibrio entre lo previsto desde el punto de vista teórico y lo que en la práctica se logra. 


\section{CONCLUSIÓN}

En la sociedad actual se necesitan mujeres y hombres democráticos y participativos, que piensen y actúen creadoramente. Los principales fundamentos en el modo de actuación creativo que deben asumir los profesionales en formación de la carrera Educación Preescolar desde el proceso de enseñanza -aprendizaje de la Disciplina Principal Integradora, la cual permite resolver las demandas sociales y dar solución a los problemas que se presentan desde su práctica profesional pedagógica. Esta disciplina garantiza el ejercicio de la profesión y la introducción del estudiante en la actividad investigativa como vía esencial para solucionar problemas inherentes a la misma de modo creativo.

\section{BIBLIOGRAFÍA}

1. Addine Fernández, Fátima (2013). La Didáctica General y su enseñanza en Educación Superior Pedagógica. La Habana. Editorial Pueblo y Educación.

2. Alarcón, R. (2015). Las Ciencias de la Educación en una Universidad integrada e innovadora. Conferencia en Congreso Internacional Pedagogía 2015. La Habana, Cuba.

3. Betancourt, J. (1997). La creatividad y sus implicaciones. ¿Por qué, para qué y cómo alcanzar la calidad? Editorial Academia, La Habana.

4. Brito, S.Y. (2015). La autoevaluación de las carreras universitarias. Tesis en opción al grado científico de Doctor en Ciencias Pedagógicas. Universidad de Sancti Spíritus José Martí Pérez, Cuba.

5. Chivás Ortíz, F. (1992). Creatividad+dinámica de grupo= ¿eureka? Editorial Pueblo y Educación. La Habana.

6. Hill, G. (1987) Orientación escolar y vocacional. Editorial. Pax. México.

7. Horruitiner, P. (2009). La universidad cubana: modelo de formación. Editorial Universitaria del Ministerio de Educación Superior. La Habana.

8. Kuliutkin. (1986) El pensamiento creador en la actividad pedagógica profesional del maestro, en: Cuestiones de Psicología, No. 2, 1986, p.21.

9. Klinberg, Lothar (1970). Introducción a la Didáctica General. Editorial Pueblo y Educación. La Habana.

10. Logan, L (1980) Estrategias para una enseñanza creativa, Barcelona, España.

11. Luz y Caballero, J. (1952). Elencos y discursos académicos.

12. Mehlhorn, G. y otros. (1982) El pensamiento creador y la actividad creadora de los estudiantes en: Educación Superior Contemporánea. No. 3, La Habana.

13. Ministerio de Educación. (2010). Modelo del Profesional de la Carrera Licenciado en Educación, Educación Preescolar. Material en Soporte Digital.

14. Ministerio de Educación Superior. (2016) Programa de Disciplina Principal Integradora. Material en Soporte Digital.

15. Mitjans. A. (1995). Pensar y crear. Estrategias, métodos y programas. Editorial Académia, La Habana.

16. Parra Vigo, Isel. B. (2005). La dirección de la competencia didáctica en la formación inicial del profesional de la educación. En Profesionalidad. La Habana. En soporte digital.

17. Remedios, J.M., Alfonso, M., Valdés, M.B., Trujillo, N. A., Hernández, T., Palau, C.M.... Clarice, V. (2016). Informe final: Sistematización acerca de la Pedagogía como ciencia de la educación Proyecto: Acercamiento teórico-metodológico a problemas de la Pedagogía Cubana. (Soporte digital)

18. Rico Pilar (1996) Reflexión y aprendizaje en el aula. Editorial. Pueblo y Educación.

19. Rojas Hernández M. (2016) Modelo de actuación de los docentes de las carreras pedagógicas para la labor educativa. Tesis en opción al grado científico de Doctor en Ciencias Pedagógicas. UNISS. "José Martí Pérez". Cuba.

20. Rojas, M. y Hernández, T. (2015). El docente universitario del siglo XXI y su función orientadora. Ponencia presentada a la VII Conferencia Científica Internacional Universidad y Sociedad, Holguín, Cuba.

21. Vigotsky, L.S. (1987). Imaginación y creación en la edad infantil. Editorial. Pueblo y Educación, La Habana. 